$\xi_{\mathrm{\beta}}=$

\title{
Application of Relief-Point Brile Font in the System of Ornamental Decoration
}

\author{
Yu.I. Bratisheva ${ }^{1 *}$, O.A. Zyabneva ${ }^{2}$, I.A.Oranskaya ${ }^{3}$ \\ * MIREA - Russian Technological University, Moscow, Russia
}

\begin{abstract}
The present article addresses the issue of psychological adaptation of environment for people with disabilities. The use of braille boldface as an ornamental design of environmental objects, which facilitates the exchange of information between people with different capabilities and the creation of emotional comfort for each person, is proposed.
\end{abstract}

Keywords: Design application, psychological adaptation, Environmental Effects,

\section{Introduction}

The most important problem in the process of environment adaptation for people with disabilities are social barriers. The idea of equal rights for all people, regardless of their special abilities, currently exists as a program, but not in the minds of the whole society. The modern world is impetuous and impulsive, and the existing programs of social protection of people with disabilities are not rarely perceived as "awkward" and "constraining." There is a problem of psychological distance, not understanding the special needs of people with disabilities [4].

Solving these problems in the field of design in the field of production and processing of materials for the formation, creation of affordable and comfortable - from the position of functioning and from the position of aesthetics - the objective human habitat [1], a gradual search for points of contact between the world of "healthy people" and the world of people with limited opportunities."

Part of the information when communicating people is transmitted through non-verbal means. Non-verbal communication is inextricably linked with speech communication and occupies an extremely important place in it.

There are achievements and cultural traditions of the world of people with disabilities associated with alternative means of communication. One such achievement is a tactile way to transfer sounds - braille, designed for visually impaired people [3]. Braille text consists of letters, numbers, and different characters, each of which are three rows of convex points [2]. Also in this font you can make different graphics and drawings. This font has become a universal way of knowing and communicating for visually impaired people.

However, there is an aspect that was not considered; it is the aesthetics of Braille, where the font is understood as an aesthetic value and an important part of the artistic and communicative system of visual culture, forming the optimal visual environment for people of different possibilities.

\section{Proposed decision}

For people with ordinary vision, Braille can be perceived as a simple geometric pattern, with its own rhythm and meaning. In the process of creating a project with an ornamental composition in Braille type, the designer must solve a number of constructive and aesthetic tasks: build the structure of the composition, identify accents, take into account psychophysiological and emotional associations [5]. Such encrypted ornaments can be used in any interior and on any objects; a message, a picture or just a name can be hidden. Figure 1 shows a sample design of a utilitarian product (mugs) using Braille in a decorative design.

Individual communication with social networks to achieve and recognize the preferences and preferences of a person in the friendship relationship to achieve this will undoubtedly have the quality of the proposer system. The collaborative filtering clustering method is presented in two steps online to an active user, a N-top recommendation list, and an offline stage where active users are divided into different clusters. The Fuzzy C-Mean (FCM) algorithm ensures the most accurate advice, the data used represents personal information and information about user profiles that are extracted through the use of user interfaces (APIs) embedded in social networks. Is. The proposed clustering method based on fuzzy logic and using the particle swarm optimization algorithm is well suited for uncertainty and has been able to cluster the same users more accurately using clustering of relative and appropriate data, which this leads to increased accuracy and error reduction compared to the user-centered and item-centered participatory filtering method.

The need to build new administrative, residential, educational and health buildings and a tendency to use energy equipment has led to an increase in energy consumption in this sector and at present, energy consumption in the construction and home sector is about $38 \%$ of the total energy consumption in the country. is. One of the most principled methods of solving the problems of energyproducing and consuming systems, the dependence on nonrenewable or renewable energies, the use of systems and control and management strategies in buildings, these elements and elements in a comprehensive manner, with the sole assumption 
that Buildings are thermodynamic systems that can be analyzed, and this new interdiscipline, which depends on different engineering sciences, is on the agenda of international scholars.

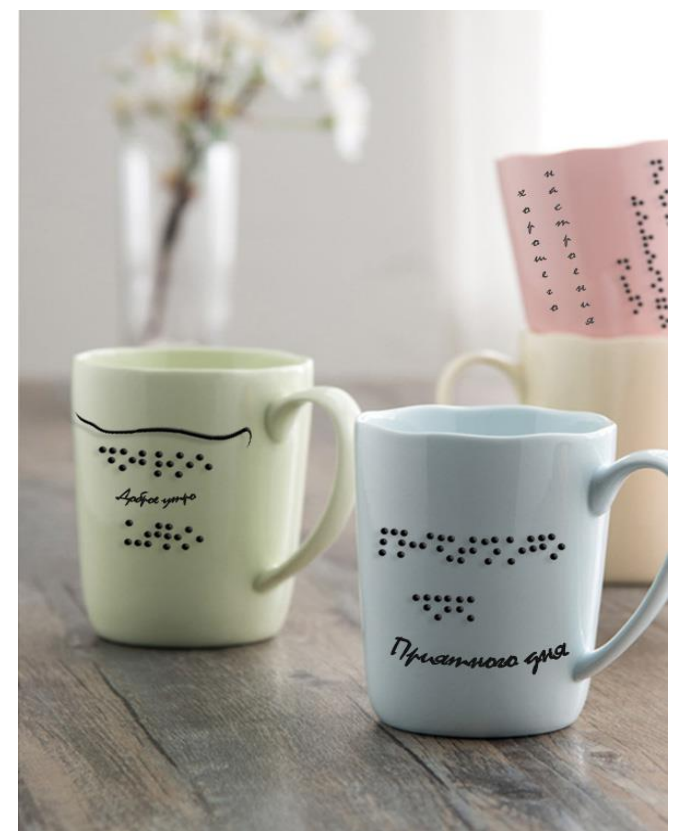

Figure 1: Application of Braille on the subject of everyday life

The decoration of the mug is universal. The information contained in it is suitable for people with any vision. Such a product is a concern for the psychological comfort of a person. For a visually impaired person, this is a source of positive information. For an ordinary person, the aesthetics of such a subject causes positive visual and tactile perceptions. Each person will reflexively, will come into contact with inscriptions, and will become familiar with the method of tactile perception of information. In Figure 2, Braille is used as an ornament on the decorative panels of the doors of the cabinet. Three Japanese hockey poems are written: So cries a pheasant, So easy, easy Here came the moon, As if it was he who opened It came out - and in the cloud And the smallest bush

The first star. Thought the moon. Invited to the holiday.

In this case, the designer deliberately did not include a graphic font, leaving a riddle in this work that can be comprehended by any modern person.

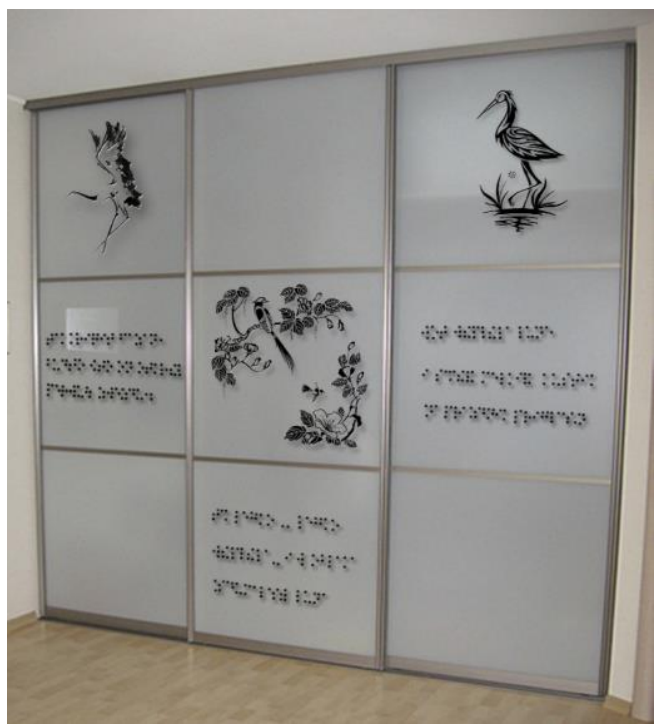

Figure 2: The use of Braille in the decoration of the interior of the house
The house is a homey and very beautiful home that does not have the potential to become a part of it and the house continues to live up to the standards of the past and does not provide enough comfort for its inhabitants. With a little attention and thought, this home can be transformed into a desirable place for life, without sacrificing home and its old values and questioning its architectural idea. In the project, after the initial studies, we have been trying to design a model for the interior design. Among the three approaches, 1 . Toghli as a work with rigid identity and invariance. 2. The interaction and creation of a kind of relationship between today and yesterday; and 3. A design with a greater tendency toward a new and contemporary identity; second approach as a balanced approach; It is suitable to preserve the historical identity of the building as well as to make it possible to use it and to use its potential. The plan of revitalization is to be reviewed by the plan, taking into account and respecting the main idea of the architect, as well as manipulating so that the abandoned spaces can be used with maximum and optimal use. The board was produced and the interior layout is also introduced by manual sketches. The research is of applied type and it analyzes the qualitative analysis of the subject by correlation and multi-variable analysis of the subject. And the necessary materials were obtained through field studies and library studies, and from the home page as a case study, the results of the research were used.

\section{Conclusion}

Thus, in the process of creating expressive visual images of aesthetic value, the use of Braille as an ornamental design contributes to the psychological adaptation of people with disabilities in society. We have incorporated the culture of visually impaired people into our daily lives and the tactile Braille system is an integral part of the global culture. This direction serves to bring together the worldviews and common feelings of all people.

\section{References}

[1] Sokolova M.L. "Modern problems of the direction" Technology of artistic processing of materials "The electronic network publication" Russian technological journal "Russiantechnologicaljournal" rtj.mirea.ru/journalarchive/2017/volume-5-no-1/

[2] Personal site. Braille -http: //specposobie.narod.ru/index/0-27

[3] People with disabilities and special needs-http: //www.tepka.ru/Obschestvoznanie_5/5.html

[4] People with disabilities. Help people with disabilities. - http://vsetemu.org/new-lyudi-s-ogranichennymi-vozmozhnostyamipomoshh-lyudyam-s-ogranichennymi-vozmozhnostyami.html

[5] Petrovsky D.I. Visible verb. Book 1. Harmony and illusions: Introduction to font arts, St. Petersburg: Chemizdat - $168 \mathrm{p}$.

[6] Kazachkova, O.A., Zyabneva, O.A., Mamedova I.Y., Kulishova E.A. 3D technologies of jewelry with elements of complicated design. International Journal of Engineering and Technology (UAE). - Volume 7, Issue 3, 2018, Pages 155-157. 